\title{
Some properties of linguistic feature detectors*
}

\author{
PETER D. EIMAS, WILLIAM E. COOPER, and JOHN D. CORBIT \\ Brown University, Providence, Rhode Island 02912
}

\begin{abstract}
A series of experiments, using a selective adaptation procedure, investigated some of the properties of the linguistic feature detectors that mediate the perception of the voiced and voiceless stop consonants. The first experiment showed that these detectors are centrally rather than peripherally located, in that monotic presentation of the adapting stimulus and test stimuli to different ears resulted in large and reliable shifts in the locus of the phonetic boundary. The second experiment revealed that the detectors are part of the specialized speech processor, inasmuch as adaptation of a voicing detector (as measured by a shift in the phonetic boundary) occurred only when the voicing information was presented in a speech context. In the third experiment, the detector mediating perception of the voiced stops was shown to be more resistant to adaptation than the detector mediating perception of the voiceless stops.
\end{abstract}

In a recent study that used a selective adaptation procedure, Eimas and Corbit (1973) presented evidence for the existence of linguistic feature detectors which mediate the perception of voicing contrasts and which are analogous to the complex feature detectors found in the visual system of man (Blakemore \& Campbell, 1969; Blakemore \& Sutton, 1969). These linguistic feature detectors are each sensitive to a different, relatively narrow range of values along the dimension of voice onset time (VOT). Variations in VOT alone are sufficient for the perceived distinctions between the voiced and voiceless stop consonants of English in initial position, that is, between [b] and [p], [d] and [t] , and [g] and [k] (Lisker \& Abramson, 1970). ${ }^{1}$ It is of importance to note that variations in VOT distinguish voicing contrasts in numerous diverse languages in addition to English (Lisker \& Abramson, 1964).

After selective adaptation with a synthetic voiced or voiceless stop, there were large and consistent alterations in the manner in which listeners identified and discriminated series of synthetically produced speech patterns that varied only in VOT. For example, after adaptation with the voiceless bilabial stop $[\mathrm{p}]$, the locus of the phonetic boundary for a series of bilabial $([b, p])$ or apical $([\mathrm{d}, \mathrm{t}])$ stop consonants shifted toward the voiceless end of the continuum, indicating that a greater number of identification responses belonged to the voiced or unadapted category. This effect was most marked for stimuli near the original phonetic boundary. Adaptation with a voiced stop produced a greater number of voiceless identification responses and a marked shift in the phonetic boundary toward the voiced end of the continuum. Finally, after adaptation with $[p]$, the peak in the bilabial discriminability function at the region of the original phonetic boundary

\footnotetext{
*We wish to thank F. S. Cooper for generously making available the facilities at the Haskins Laboratories. This research was supported by PHS Grants HD 05331 and MH 16608, as well as by an NSF Undergraduate Research Participation Fellowship from the Department of Linguistics. Harvard University, to W.L.C.
}

shifted such that it.now corresponded with the locus of the phonetic boundary after adaptation.

Inasmuch as adaptation systematically affected the discriminability function and, moreover, produced an approximately equivalent effect on the identification functions regardless of whether the adaptating stimulus and identification series were from the same class of consonants, it is highly unlikely that these effects were due to adaptation of the sound pattern as an entire phonetic unit. Were this the case, it would be difficult to explain how a change in the percept of a bilabial stop would alter the perception of a series of apical stops or, for that matter, alter the form of the bilabial discriminability function. A more realistic explanation of these data is that there exist two feature detectors, each of which is especially tuned to a restricted range of VOT values and mediates the perception of one of the two categories of voicing. Selective adaptation functions to lower the sensitivity of a feature detector, thereby altering both the identification and discrimination functions, the latter of which has been shown to be dependent upon the ability of listeners to assign differential phonetic labels to the stimuli (Liberman et al, 1967).

The present set of experiments was directed toward obtaining additional information concerning the nature of these linguistic feature detectors. We were concerned first with the location of these detectors-that is. whether they were central or peripheral analyzers of acoustic information. A second and related question was whether these detectors were part of the specialized speech processing system or whether they existed as part of the more general auditory perceptual apparatus. Finally, we were concerned with investigating further the earlier finding that the detector mediating perception of the voiced stops was more resistant to the effects of selective adaptation than was the detector underlying perception of the voiceless stops.

\section{EXPERIMENT I}

This experiment was designed to determine the locus 
Table 1

Individual and Mean Loci of the Phonetic Boundaries in Milliseconds With and Without Adaptation With the Voiceless Stop [t]

\begin{tabular}{lccc}
\hline Ss & $\begin{array}{c}\text { Without } \\
\text { Adaptation }\end{array}$ & $\begin{array}{c}\text { Binaural } \\
\text { Adaptation }\end{array}$ & $\begin{array}{c}\text { Monotic } \\
\text { Adaptation }\end{array}$ \\
\hline 1 & 40.0 & 49.0 & 43.8 \\
2 & 32.7 & 39.0 & 38.1 \\
3 & 30.8 & 45.3 & 45.8 \\
4 & 41.3 & 58.9 & 61.3 \\
5 & 37.1 & 48.5 & 47.3 \\
Mean & 36.4 & 48.1 & 47.3 \\
\hline
\end{tabular}

of the VOT detectors. In order to make this determination, several measures of the site of the phonetic boundary were necessary. First, the phonetic boundary for a series of apical stops was determined for listeners in an unadapted state. Next, estimation of the boundary was made after selective adaptation, in this instance, with the voiceless apical stop [t]. For these first two measures of the phonetic boundary, the stimuli were presented binaurally, as in the original study. Finally, the effects of adaptation on the phonetic boundary were measured when the adapting stimulus (again [t]) and the stimuli to be identified were presented monotically, with the adapting stimulus presented to one ear and the stimuli to be identified to the other ear. If adaptation occurs under monotic conditions, then the detectors must be central rather than peripheral.

\section{Method}

\section{Stimuli}

The stimuli were a series of 14 synthetic speech patterns, produced by means of a computer-controlled parallel resonance synthesizer by Lisker and Abramson (1970). The stimuli, which were perceived as either the voiced or voiceless apical stops [d] or $[t]$, respectively, plus the vowel [a], varied only in VOT Variations in VOT are defined as the variations in time between the release burst and the onset of laryngeal pulsing (Lisker \& Abramson, 1964). These variations may be realized synthetically by varying the onset of the first formant relative to the onset of the second and third formants and by having the second and third formants excited by a noise source as opposed to a periodic source during the interval when the first formant is not present. Short lags in VOT are perceived as the English voiced stops, whereas relatively long lags are perceived as voiceless stops, in the present situation as [d] and [t]. The series of stimuli had VOT values from $0 \mathrm{msec}$ (voicing and the onset of the first formant simultaneous with the onset of the second and third formants) to $+80 \mathrm{msec}$ (relatively long voicing lag). The difference between successive stimuli was $5 \mathrm{msec}$, except for the final four stimuli, where the difference was $10 \mathrm{msec}_{n}$ Each stimulus was $450 \mathrm{msec}$ in duration.

\section{Procedure}

Identification functions were obtained from listeners in an unadapted state by presenting random sequences of the 14 stimuli binaurally by means of an Ampex AG-500 recorder and speaker system at a comfortable listening level. Each stimulus was presented 20 times, and the interstimulus interval was
$3.5 \mathrm{sec}$. On the day after the initial identification test, a series of six sessions was begun, during which identification responses to the same series of synthetic speech were obtained with listeners in an adapted state. The order of presentation of the different adapting stimuli was as follows: Session 1, [d] ; Session 2, [t] ; Session 3, bqth [d] and [t], the two stimuli being Cpresented alternatingly during each individual adaptation trial; Session 4 , monotically presented [t] ; Sessions 5 and 6 , the initial $50 \mathrm{msec}$ of the synthetic speech pattern used in Session 1.

The data from Sessions 2 and 4 are the concern of Experiment I. Experiment II considers the identification responses obtained from Sessions 1, 5, and 6, and Experiment III considers the data from Sessions 1, 2, and 3. It should be noted that neither the number of previous adaptation sessions nor the order of the sessions appears to alter the results, provided only that sufficient time elapses between sessions to permit full recovery from the effects of the earlier session. We have used a minimum of $24 \mathrm{~h}$ as a conservative estimate of the time necessary for recovery to be complete.

The first adaptation session of concern to Experiment I was conducted as follows. The listeners were presented at the beginning of the session with 2 min of the adapting stimulus, which had a VOT value of $+80 \mathrm{msec}$ and which was always perceived as [ $t]$ plus the vowel $[a]$. During this time, the stimulus was presented 150 times, with $350 \mathrm{msec}$ separating each presentation. Next, 35 adaptation trials were given. During each trial, the adapting stimulus was presented for $1 \mathrm{~min}(75$ repetitions) followed by a $2-\mathrm{sec}$ interval of silence and then four randomly selected stimuli from the series of 14 apical stops. Each stimulus was identified as [d] or [t] immediately after presentation during the 2 -sec interval between stimuli. After the last stimulus to be identified was presented, a 5 -sec interval of silence occurred before the start of the next adaptation trial. In this manner, each stimulus was presented 10 times for identification. In this session, all stimuli were presented binaurally. In the second adaptation session of concern, exactly the same general procedure was used, except that the stimuli were presented to the listeners monotically rather than binaurally. For two of the listeners, the adapting stimulus was presented to the right ear and the stimuli to be identified to the left ear. This was accomplished by means of high-quality stereo ear phones (Koss Pro 600A). The opposite ear arrangement was used for the remaining three listeners. Each of the two adaptation sessions required approximately $1 \mathrm{~h}$, which included short breaks.

\section{Subjects}

The five listeners, all paid volunteers, were undergraduate students at Brown University. Each had participated in at least the first four adaptation sessions.

\section{Results and Discussion}

The results of Experiment $\mathrm{I}$ are shown in Table 1. Herein are presented the individual and mean phonetic boundaries in milliseconds for the three experimental conditions: two adaptation sessions and one identification session without adaptation. The effect of binaural adaptation with the voiceless stop [t] was virtually the same as that obtained in the original study: the mean shift in the phonetic boundary (unadapted estimate of the phonetic boundary minus the estimate of the phonetic boundary after adaptation) was $-11.7 \mathrm{msec}$, whereas the comparable figure from the original study was $-11.5 \mathrm{msec}^{2}$ As in the original study, for each listener a greater number of identification 
responses belonging to the voiced category occurred after adaptation, especially for those stimuli near the unadapted phonetic boundary. After adaptation, when the adapting stimulus and the identified stimuli were presented monotically to different ears, the mean shift in the boundary was -10.9 msec. Both adaptation sessions resulted in a reliable shift in the boundary $(p<.01)$, whereas the difference between the two adaptation sessions was not statistically significant. ${ }^{3}$

An examination of the individual data revealed no consistent differences between the two adaptation tests; compared with binaural adaptation, the magnitude of the phonetic boundary shift after monotic adaptation was greater in two listeners and smaller in three listeners, and in only one instance was the difference between sessions greater than $2.3 \mathrm{msec}$. In summary, the results unequivocally support the contention that the dimension of VOT is analyzed or detected beyond the level of a peripheral analyzing system. In other words, the detectors for VOT are part of a central processing system. Whether they are also a part of the specialized speech processing system was the subject of the next experiment.

\section{EXPERIMENT II}

In order to determine whether the analysis of VOT is undertaken by a general auditory processor or a specialized speech processor, we compared the effects of selective adaptation under two conditions: (a) when the voicing information was presented in a speech context, and (b) when the voicing information was presented in a nonspeech context. The former was achieved by repeatedly presenting a good exemplar of the voiced stops, in this experiment a synthetic speech pattern with a VOT value of $0 \mathrm{msec}$, which was perceived by all listeners as [d] plus the vowel $[a]$. To realize the latter condition, listeners were repeatedly exposed to the initial $50 \mathrm{msec}$ of the synthetic voiced stop described above. The initial portion of the pattern is not perceived as speech by most listeners and was. in fact, not perceived as speech by any of our listeners. Reports of the listeners' perceptions included the following descriptions: "water dripping," "dull hammering," and "just a noise." The reason for the nonspeech quality of the shortened synthetic pattern (referred to hereafter as d-chirp) undoubtedly derives from the fact that some of the necessary acoustic information for consonantal identification is carried by the vowel or steady-state portion of a $\mathrm{CV}$ syllable. This information was entirely eliminated in constructing the d-chirp. (For a more complete description of the cues for consonantal perception. see Liberman et al, 1967.)

It was the case. of course, that the d-chirp version of the adapting stimulus contained the same VOT information as did the speech-like form of the same stimulus. A shift in the locus of the phonetic boundary with the speech pattern as the adapting stimulus and the absence of a shift with the d-chirp would permit the inference that the detectors for VOT are part of the speech processing mechanism and not a property of the more general auditory system.

\section{Method}

\section{Subjects}

The listeners were seven paid volunteers, all of whom were Brown University undergraduates. Four Ss had had no previous experience with synthetic speech. Two of the three experienced listeners had undergone all six of the adaptation sessions, described in Experiment I, while the third listener underwent only five adaptation sessions, having missed the session with the monotically presented $[t]$.

\section{Stimuli}

The stimuli were the same series of 14 synthetic speech patterns used in Experiment I. They were all perceived as [d] or [t] by the seven Ss. The speech-like adapting stimulus had a VOT value of $0 \mathrm{msec}$ (voicing and the first formant begin simultaneously with the onset of the second and third formants). The d-chirp was constructed by removing the final $400 \mathrm{msec}$ from the speech-like adapting stimulus. Later spectrographic analyses showed that the duration of the d-chirp was 45 to $50 \mathrm{msec}$ in each instance.

\section{Procedure}

Each of the four naive listeners participated in four daily sessions, each of which permitted an estimate of the locus of the phonetic boundary. The order in which the four estimates were made was as follows: (1) in an unadapted state, (2) after adaptation with d-chirp, (3) after adaptation with [d], and (4) again after adaptation with d-chirp. For the three experienced listeners, disregarding the adaptation sessions not relevant to the present experiment, the order of sessions was 1 . 3,2 , and 4 . Just prior to the second session with the d-chirp, the listeners were informed as to be the nature of the stimulus and instructed to try to hear the stimulus as speech, that is, as [d]. The purpose of this session was to attempt to induce analysis of the adapting stimulus by the speech processor.

The manner in which the identification functions were obtained. whether with or without adaptation, was the same as described in Experiment I. The only change in the procedure was that different random orders of the stimuli to be identified were used in each of the adaptation sessions. Each session again lasted for approximately $1 \mathrm{~h}$, and the stimuli were all presented binaurally.

\section{Results and Discussion}

The individual and mean phonetic boundaries for each of the four sessions are shown in Table 2. Again, the results after adaptation with the entire synthetic pattern are strikingly similar to those found by Eimas and Corbit (1973): the mean boundary shift was $6.9 \mathrm{msec}$ in the present study and $7.8 \mathrm{msec}$ for the comparable condition in the original study. All seven listeners showed the predicted adaptation effect, in that after adaptation with a voiced stop. there was a greater number of voiceless identification responses. After adaptation with the d-chirp. with or without instructions to perceive the stimulus as speech. statistically. 
Table 2

Individual and Mean Loci of the Phonetic Boundaries in Milliseconds Without Adaptation and After Adaptation With [d] and [d]-Chirp

\begin{tabular}{lcccc} 
& \multicolumn{2}{c}{ Adaptation } & With [d] & and [d] Chirp \\
\hline Ss & $\begin{array}{c}\text { Without } \\
\text { Adaptation }\end{array}$ & [d] & \multicolumn{2}{c}{$\begin{array}{c}\text { Adaptation With } \\
\text { [d] -Chirp [d] -Chirp* }\end{array}$} \\
\hline 1 & 43.2 & 36.2 & 45.0 & 42.3 \\
2 & 32.7 & 19.1 & 29.5 & 35.0 \\
3 & 31.7 & 27.1 & 30.9 & 34.5 \\
4 & 32.9 & 30.4 & 30.4 & 30.6 \\
5 & 32.7 & 28.3 & 31.8 & 29.8 \\
6 & 41.3 & 36.4 & 38.9 & 38.3 \\
7 & 25.1 & 13.7 & 26.5 & 17.0 \\
Mean & 34.2 & 27.3 & 33.3. & 32.5 \\
\hline
\end{tabular}

*With instructions to hear the adapting sound as speech.

nonsignificant changes in the boundary were obtained. The mean phonetic boundary after adaptation with the entire synthetic pattern differed reliably from the mean boundaries obtained without adaptation and with adaptation with the d-chirp ( $p<.05$ in each instance).

Examination of the individual data revealed that only one listener showed essentially the same magnitude of boundary shift under all three adaptation conditions. It is perhaps worthy of note that this same listener showed the smallest boundary shift. Likewise, only one listener showed a boundary shift greater than $3 \mathrm{msec}$ with the d-chirp adapting stimulus, and this interestingly occurred after instructions to hear the sound as speech. However, to conclude that it is possible to induce processing in a speech mode by instruction is at best tenuous, given the single instance of the effect.

We partially replicated this experiment, using the voiceless apical stop [t] and the initial $50 \mathrm{msec}$ of the same pattern as adapting stimuli. Phonetic boundaries for the series of 14 apical stops were determined for four additional naive listeners when they were in an unadapted state, after adaptation with the entire synthetic pattern, and after adaptation with the t-chirp. The mean shift in the locus of the phonetic boundary was $9.0 \mathrm{msec}$ after adaptation with the entire pattern and $.3 \mathrm{msec}$ after adaptation with the chirp-like sound. The difference was reliable at better than .01 level of significance.

The inference permitted by these data is that VOT detectors are part of the speech processing system. Moreover, in order for the speech processing mechanism to be activated, there must be some minimum of acoustic information signaling the presence of speech. It is apparent that the presence or absence of voicing and rapidly changing formant transitions are not sufficient in and of themselves for activation of the speech processing mechanism (cf. Mattingly et al, 1971).

\section{EXPERIMENT III}

This experiment further investigated the finding (Eimas \& Corbit, 1973) that the feature detector underlying perception of the voiced stops was more resistant to the effects of selective adaptation. This is, adaptation with a voiced stop produced a smaller shift in the phonetic boundary than adaptation with a voiceless stop. As in the original study, we compared the magnitude of the boundary shift after selective adaptation with a voiced stop with that obtained after adaptation with a voiceless stop. In addition, the direction and extent of shift in the phonetic boundary was measured after adaptation of both detectors simultaneously. This was accomplished by the repeated presentation of both [d] and [t] in an alternating sequence. By means of this procedure, it is possible to obtain a second estimate of the relative resistance of these detectors to adaptation, as well as to determine whether the effects of adapting both detectors at the same time are additive. For example, if after selective adaptation with [d] and then [t] there is a $+5-\mathrm{msec}$ and a $-10-\mathrm{msec}$ shift in the phonetic boundary, respectively, then after simultaneous adaptation of both detectors, the expectation from the additivity hypothesis is for a -5 -msec shift in the boundary.

\section{Method}

\section{Subjects}

The listeners were seven paid volunteers, all of whom were undergraduates at Brown University. Each $O$ had participated in at least the first three adaptation sessions outlined in Experiment I.

\section{Stimuli}

The stimuli were the series of 14 synthetic apical stops, described in Experiment I. The two adapting stimuli had VOT values of 0 and $+80 \mathrm{msec}$, the voiced and voiceless stops, respectively.

\section{Procedure}

Estimates of the phonetic boundaries relevant to Experiment III were obtained from four experimental sessions. In the first session, all listeners were in an unadapted state. The procedural details are described in Experiment I. In the second and third sessions, listeners were selectively adapted with [d] and then $[t]$. Again, the procedural details may be found in Experiment I. In the final session, both detectors were adapted by repeatedly presenting both [d] and [t] in an alternating pattern. The only changes in procedure were that at the beginning of the session, the two adapting stimuli were presented for $4 \mathrm{~min}$ rather than $2 \mathrm{~min}$ (150 presentations of each stimulus), and that on each adaptation trial, the adapting stimuli were presented for $2 \mathrm{~min}$ rather than $1 \mathrm{~min}$ ( 75 presentations of each stimulus). On each trial, the initial adapting stimulus, [d] or $[t]$, was randomly determined.

\section{Results and Discussion}

Table 3 shows the individual and mean phonetic boundaries for each of the four sessions. For all listeners, the effects of selective adaptation were as expected. The mean shift after adaptation with [d] was $6.9 \mathrm{msec}$ $(\mathrm{p}<.01)$ and after adaptation with $[\mathrm{t}],-13.7 \mathrm{msec}$ 
$(p<.01)$. The difference of $6.8 \mathrm{msec}$ was likewise reliable $(p<.05)$. After adaptation of both detectors, there was a greater number of voiced identification responses, indicating, as in the selective adaptation sessions, that the detector mediating perception of the voiced stops was more resistant to adaptation. The mean shift in the phonetic boundary was $-4.3 \mathrm{msec}(\mathrm{p}<.05)$. In six of the seven listeners, greater adaptation effects were found for the detector underlying the voiceless stops, whether after selective or simultaneous adaptation. The single exception in both instances was the same listener.

Table 4 presents the obtained and predicted shifts in the phonetic boundary after simultaneous adaptation of both detectors. The predicted estimates are derived from the additivity hypothesis using the data from the two selective adaptation sessions. Deviations between obtained and predicted shifts are also shown in Table 4 . The mean deviation is relatively small, approximately $2.5 \mathrm{msec}$. However, the individual deviations are rather large in most cases and, moreover, inconsistent in the direction of their discrepancy. Hence, it appears that there is little support for the additivity hypothesis in the present data.

In a similar study, eight naive Ss had the adapting stimuli ([b] and [p] with VOT values of -10 and $+60 \mathrm{msec}$ ) presented dichotically: the voiced stop to the left ear and at the same time the voiceless stop to the right ear for half of the listeners, and conversely for the remaining Ss. Identification functions were obtained for two series of 14 synthetic speech patterns presented binaurally: one series signaled the apical stops and the other signaled the velar stops $([\mathrm{g}, \mathrm{k}])$. The mean shifts in the phonetic boundary after adaptation of both detectors were $-5.2 \mathrm{msec}$ for the apical stops and $-2.0 \mathrm{msec}$ for the velar stops. These data, while again demonstrating greater adaptation of the detector for voiceless stops, also indicate that adaptation effects are not dependent upon the adapting stimuli and identification series belonging to the same consonantal class. The latter finding, also obtained in the original study, is strong evidence against interpretations based on simple contrast effects or adaptation of the speech pattern as an entire phonetic unit.

Table 3

Individual and Mean Loci of the Phonetic Boundaries in Milliseconds Without Adaptation and After Adaptation With $[d],[t]$, and [d] and [t] in the Same Session

\begin{tabular}{ccccc}
\hline & Without & \multicolumn{3}{c}{ Adaptation With } \\
Ss & $\begin{array}{c}\text { Adaptation } \\
{[\mathrm{d}]}\end{array}$ & {$[\mathrm{d}]$ and $[\mathrm{t}]$} \\
\hline 1 & 40.0 & 30.8 & 49.0 & 35.0 \\
2 & 32.7 & 28.3 & 39.0 & 38.1 \\
3 & 33.6 & 25.9 & 58.5 & 40.0 \\
4 & 30.8 & 29.6 & 45.3 & 39.2 \\
5 & 41.3 & 36.4 & 58.9 & 46.2 \\
6 & 25.1 & 13.7 & 37.2 & 32.3 \\
7 & 37.1 & 27.7 & 48.5 & 40.2 \\
Mlean & 34.4 & 27.5 & 48.1 & 38.7 \\
\hline
\end{tabular}

Table 4

Predicted and Obtained Shifts in the Phonetic Boundary After Adaptation With Both [d] and [t] (in Milliseconds)

\begin{tabular}{lccc}
\hline Ss & $\begin{array}{c}\text { Obtained } \\
\text { Shifts }\end{array}$ & $\begin{array}{c}\text { Predicted } \\
\text { Shifts }\end{array}$ & $\begin{array}{c}\text { Obtained } \\
\text { Minus } \\
\text { Predicted }\end{array}$ \\
\hline 1 & +5.0 & +.2 & +4.8 \\
2 & -5.4 & -1.9 & -3.5 \\
3 & -6.4 & -17.2 & +10.8 \\
4 & -8.4 & -13.3 & +4.9 \\
5 & -4.9 & -12.7 & +7.8 \\
6 & -7.2 & -.7 & -6.5 \\
7 & -3.1 & -2.0 & -1.1 \\
Mean & -4.3 & -6.8 & +2.5 \\
\hline
\end{tabular}

\section{SUMMARY AND CONCLUSIONS}

The results of the present studies provide additional evidence for the existence of linguistic feature detectors that mediate the perception of voicing contrasts. Moreover, these data indicate that the voicing detectors are part of the central speech processing mechanism which functions to analyze not only semantic and syntactic information (Geschwind, 1970) but also phonetic information (Studdert-Kennedy \& Shankweiler, 1970).

With regard to the finding that the detector for short voicing lags (that is, for voiced stops) is more resistant to adaptation, it is of interest to note two additional facts connected with this voicing distinction. First, Lisker and Abramson (1964) found that short voicing lags were present in the productions of all 11 languages that they studied, whereas the use of long voicing lags (as in Thai) or relatively long voicing lags (as in English), or both, was optional. Second, Port and Preston (1972) have shown that it is this voicing category (short voicing lag) that is first differentiated in the utterances of young children. Thus, the primacy and possible universality with respect to the production of voiced stops is matched on the perceptual side by a detector that is more resistant to disruption. This, together with the more general finding that there exist detectors that are finely tuned to the acoustic consequences of production, is, we believe, strong corroborative evidence for the very close relationship between the processes of speech production and speech perception.

\section{REFERENCES}

Blakemore, C., \& Campbell. F. W. On the existence of neurons in the human visual system selectively sensitive to the orientation and size of retinal images. Journal of Physiology. 1969. 203, 237-260.

Blakemore, C., \& Sutton. P. Size adaptation: A new aftereffect. Science, 1969, 166. 245-247.

Eimas. P. D., \& Corbit. J. D. Selective adaptation of linguistic feature detectors. Cognitive Psychology. 1973, 4. in press.

Geschwind. N. The organization of language and the brain. Science. 1970. 170. 940-944.

Liberman. A. M.. Cooper. I. S.. Shankweiler. D. P.. \& 
Studdert-Kennedy, M. Perception of the speech code. Psychological Review, 1967, 74, 431-461.

Lisker, L., \& Abramson, A. S. A cross-language study of voicing in initial stops: Acoustical measurements. Word, 1964, 20 , 384-422.

Lisker. L., \& Abramson, A. S. Some effects of context on voice onset time in English stops. Language \& Speech, 1967, 10, $1-28$.

Lisker, L., \& Abramson, A. S. The voicing dimension: Some experiments in comparative phonetics. In Proceedings of the Sixth International Congress of Phonetic Sciences, Prague, 1967. Prague: Academia, 1970. Pp. 563-567.

Mattingly, I. G., Liberman, A. M., Syrdal, A. K., \& Halwes, T. Discrimination in speech and nonspeech modes. Cognitive Psychology, 1971, 2, 131-157.

Port, D. K., \& Preston, M. S. Early apical stop production: A voice onset time analysis. Status Report on Speech Research, SR-29/30, Haskins Laboratories, New Haven, Connecticut, 1972. Pp. 125-149.

Studdert-Kennedy, M., \& Shankweiler, D. Hemispheric specialization for speech perception. Journal of the Acoustical Society of America, 1970, 48, 579-594.

\section{NOTES}

1. Although we have limited our research to voicing distinctions in absolute initial position, where VOT is relatively insensitive to context (Lisker \& Abramson, 1967), it should be noted that VOT effectively separates the voiced and voiceless stop categories in continuous speech (Lisker \& Abramson, 1964).

2. The phonetic boundaries in all instances were estimated from the equation $z=a+b$ (VOT), when $z$ was set equal to zero. The two parameter values were determined by a least-square solution. It is of some interest to note that the slopes of the identification functions were not greatly affected by the experimental treatments.

3. In all instances, the statistical significance of shifts in the phonetic boundary was determined by $t$ tests for correlated measures.

(Revision received for publication November 25, 1972.) 